\title{
A Low-Cost ZigBee-based Wireless Industrial Automation System
}

\author{
Pedro Castro $^{1}$, João L. Afonso ${ }^{2}$, and José A. Afonso ${ }^{1}$ \\ ${ }^{1}$ CMEMS-UMinho R\&D Center, University of Minho, 4800-058, Guimarães, Portugal \\ a55615@alunos.uminho.pt, jose.afonsoddei.uminho.pt \\ ${ }^{2}$ Centro ALGORITMI, University of Minho, 4800-058, Guimarães, Portugal \\ joao.l.afonsoealgoritmi.uminho.pt
}

\begin{abstract}
This paper describes the development of an industrial automation system based on a ZigBee wireless sensor network, designed for the monitoring and control of multiple refrigeration equipments in an industrial area, replacing the existing cabled network, which is based on the LonWorks platform. For this purpose, ZigBee routers were used to replace the local controllers at the refrigeration equipments, while the central management controller was replaced by a ZigBee coordinator and a PC. The proposed system was developed using a hardware platform based in the CC2530 integrated circuit and the Z-Stack software. Results from experimental field tests performed in an industrial environment are provided in order to assess the performance of the developed ZigBee network.
\end{abstract}

Keywords: ZigBee, monitoring and control, wireless sensor networks.

\section{Introduction}

The increasing development in the field of telecommunications enabled, in the last decades, a marked evolution and expansion of the market of wireless network technologies, which are everywhere nowadays, due to the convenience and reduction of costs that result from the elimination of cables.

Many wireless communication technologies, such as IEEE $802.11 / \mathrm{Wi}-\mathrm{Fi}[1]$ aim to provide high data rates to the users. Others, such as ZigBee [2], were conceived to scenarios where high data rates are not relevant, but low cost, low energy consumption and small device size are paramount, being applied in the context of the wireless sensor networks (WSN) [3].

The evolution of the WSNs in the last decade, along with the progress in the area of sensors and actuators, allowed the emergence of applications if several areas, such as medicine [4,5], agriculture [6], industry [7], smart buildings [8], etc. The characteristics of WSNs are suitable to industrial environments, for monitoring and control of multiple equipments distributed in a given area. In such scenario, the network nodes are normally responsible to deliver the information acquired from the sensors to a central station, where relevant parameters can be monitored and controlled.

adfa, p. 1, 2011.

(C) Springer-Verlag Berlin Heidelberg 2011 
Besides the lower material and maintenance costs due to the elimination of cables, ZigBee allow more flexibility in the deployment and expansion of networks, even after they started operating, without requiring manual alterations to the network topology. Due to the low complexity, ZigBee devices normally have lower costs than their wired counterparts. Unlike analogous wireless technologies such as Bluetooth, ZigBee possesses multihop capabilities, which allows extending the wireless network coverage.

This paper presents a ZigBee-based wireless automation system designed to operate in industrial/commercial environments and replace the existing wired network and the respective controllers. The proposed system is applied to the monitoring and control of multiple refrigeration equipments installed in an industrial area.

The rest of this paper is organized as follows. Section 2 describes the architecture and components of the original cabled monitoring and control system. Section 3 presents the wireless industrial automation system that was developed to replace the cabled system. Section 4 presents experimental network performance results from tests performed in an industrial environment, and Section 5 presents the conclusions.

\section{$2 \quad$ Legacy Wired System}

The monitoring and control of refrigeration systems is based on a network that provides communication between the different devices. These refrigeration systems are installed in large areas such as supermarkets and hypermarkets, trading posts and factories, where there is a requirement for a large number of refrigerated places and equipments.

There are various types of refrigeration equipments, such as refrigeration chambers, display cabinets, shop windows, etc. Fig. 1 presents the architecture of a generic refrigeration equipment and the associated components, which are mostly sensors and actuators that can vary according to the function of the equipment. This refrigeration equipment is composed by an expansion valve, a fan, two temperature probes, a door sensor, an evaporator and an electric panel.

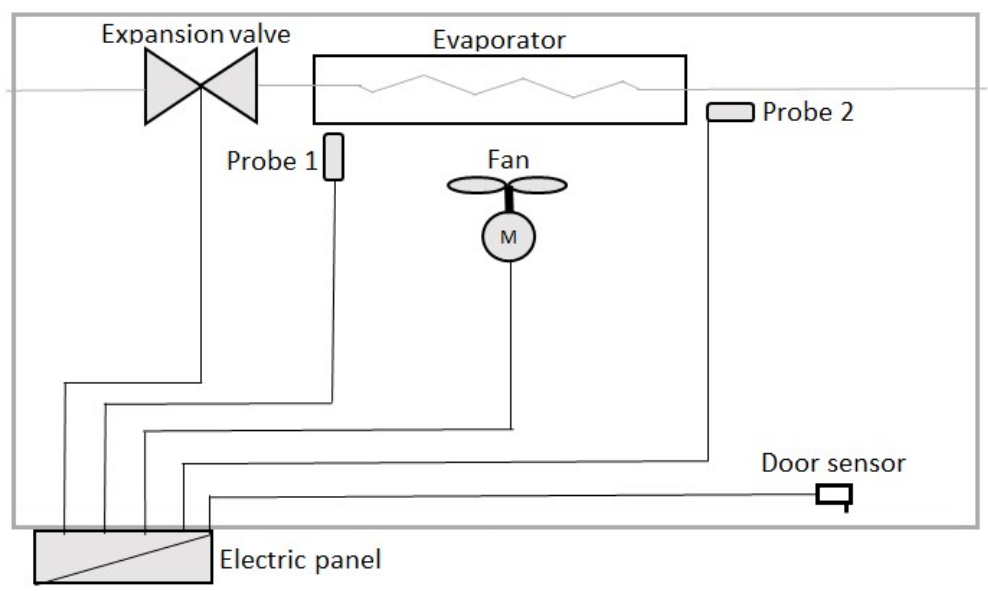

Fig. 1. Architecture of a generic refrigeration system. 
The electric panel serves as a support infrastructure for the operation of the sensors and actuators of the refrigeration system. For that purpose, it is composed by a controller and some electromechanical and thermal relays. Fig. 2 (a) shows a type of controller (AK-PC 530 [9]) that can be used. This component is responsible for the monitoring and control of the refrigeration equipment and also to communicate with a central management controller in order to send data from the sensors and the state of the actuators, as well as to receive commands from the central controller. The electromechanical relays are used on the power circuit to control the operation of the components that require currents higher than the controller is able to provide, such as the fan or the expansion valve, while the thermal relays are used to protect these power circuits.

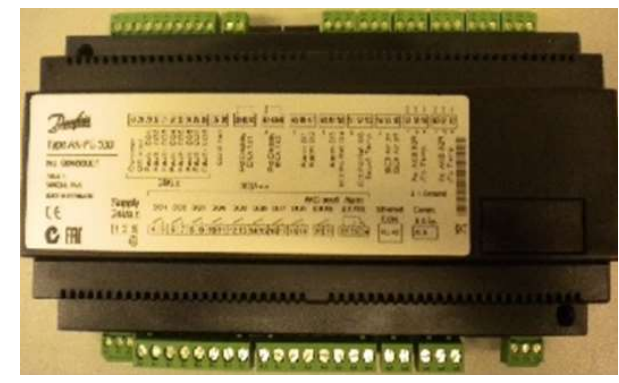

(a)

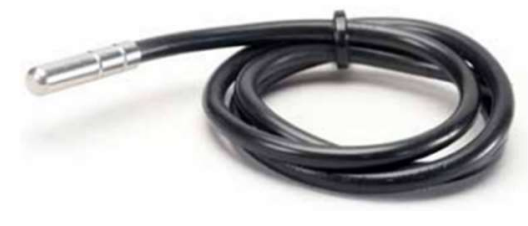

(b)

Fig. 2. Components of the original system: (a) AK-PC530 controller; (b) EKS 111 probe.

Each refrigeration equipment includes two temperature probes (probe 1 and probe 2), at specific locations. Fig. 2 (b) shows a type of probe (EKS 111 [10]) that is used. These are PTC (Positive Temperature Coefficient) probes, which mean that their electric resistance increase with the temperature. These probes are directly connected to the controller, where their analog signal is converted to digital.

The door sensor is a miniature snap-action switch (also known as a micro switch) that is installed at the refrigeration equipment door. Its function is to provide information to the controller regarding the state of the door: open or closed.

The expansion valve controls the flux of the cooling fluid, which allows the evaporator to refrigerate the equipment through thermodynamic effects. The controller on the electric panel turns this valve on or off according to the readings from the probes and the temperature values defined for the refrigeration equipment. The expansion valves used in the original system are of type AKV 10 [11], and are powered with $230 \mathrm{~V}$ AC.

The fans control the flux of air that goes through the evaporator. They are connected to the power circuit and are commanded by the controller. As in the case of the valve, they can be turned on or off, and are powered with $230 \mathrm{~V}$ AC.

A central management controller is used to monitor and control all local refrigeration equipments. This controller receives data from the controllers of all the refrigeration equipments and sends commands back using network cables. It is also responsible for the user interface. The central management controller used in the original system (AKSM 850 [12]) provides a display and access buttons to allow the user to monitor and configure the operation of the refrigeration equipments without requirement of any 
other external equipment. This controller is normally placed in the central electric panel in the machine room.

The communication between the devices of the original system is based on the LonWorks [13] network technology, and uses the standard RS-485 in the specification of the physical layer of the LonTalk protocol. The devices are connected in a bus topology using twisted pair cables. This topology allows a maximum transfer rate of $39.06 \mathrm{kbps}$ and a maximum of 60 controllers. A repeater is required when the network cable length extends beyond the maximum length defined by this network technology (1200 meters). The repeater used by the legacy system is the AKA 223.

\section{Developed Wireless System}

This work proposes the replacement of a cabled LonWorks network by a ZigBee-based wireless sensor network (WSN) satisfying similar operation requirements. The controllers are the main supporting devices for the operation of the LonWorks network. Therefore, the local controllers installed in the electric panels of the refrigeration equipments were replaced by ZigBee routers (ZR), while the central management controller was replaced by a ZigBee coordinator and a PC, which work as the central station. The cabled infrastructure between the controllers, based on a bus topology, was replaced by a ZigBee wireless network based on a mesh topology. The remaining components of the refrigeration equipments remained the same.

\subsection{ZigBee network}

The ZigBee standard defines the upper layers of the ZigBee protocol stack, including the network layer (NWK) and the application layer (APL), while the IEEE 802.15.4 standard defines the physical (PHY) and the medium access control (MAC) layers. The devices used in this system operate in the $2.4 \mathrm{GHz}$ frequency band with a PHY data rate of $250 \mathrm{kbps}$.

Since the ZigBee devices in this system have easy access to the mains power at the electric panels, it is not necessary to resort to batteries; therefore, there are no stringent restrictions in terms of energy consumption. Consequently, we decided to replace the local controllers by ZigBee routers, instead of using ZigBee end devices (ZED), which could potentially sleep during inactive periods in order to save energy. One advantage of the use of ZRs (which are distributed over the industrial facility) instead of ZEDs is that, thanks to the multihop mesh topology, they can seamless extend the coverage of the ZigBee network, relaying the packets of other ZRs that are out of the range of the ZigBee coordinator (ZC) and increasing the reliability of the network through the provision of alternative routes. Each ZR was physically connected to the sensors and actuators of the respective refrigeration equipment.

Besides the local monitoring and control of its sensors and actuators, each ZR sends data and receives the commands from the ZC located at the central electric panel, either directly or through other ZRs. It is also possible for a ZR to monitor sensors or control actuators from other refrigeration equipments through the wireless network if required. 
After the network association process, a ZR can start to transfer data to the ZC. The first data frame sent by the ZR contains the identifier of the respective refrigeration equipment (the equipment number), as well as the characteristics of the sensors and actuators it has. After that, the subsequent data frames transport readings from the sensors and the state of the actuators connected to the ZR. These frames are sent periodically each one second. They have a payload of 20 bytes, and their total length, including the ZigBee headers and trailers, is 47 bytes.

\subsection{Network hardware and software}

The hardware platform used during the development of the code for the ZigBee devices and for the experimental evaluation of the wireless network performance was the CC2530 development kit, from Texas Instruments (TI). The main component of this kit is the CC2530 [14] SoC (System on Chip), which integrates a 8051-based microcontroller and a transceiver compliant with the IEEE 802.15.4 standard in the same chip. Each network device was composed by two boards that are connected together: a CC2530EM (Evaluation Module), which contains the CC2530 and other auxiliary components required for the operation of this chip and for wireless communication; and a SmartRF05EB (Evaluation Board), which provides different alternatives to power the CC2530EM and contains several other components that are useful during the development phase, such as an LCD display, LEDs, buttons, and USB and RS-232 interfaces. After the development phase, these two boards can be replaced by smaller and lower cost alternatives.

Fig. 3 presents the central station board that was developed to be connected to a PC and act as the ZigBee coordinator (ZC) of the wireless network, replacing the much larger boards of the development kit (this board measures $24 \mathrm{~mm}$ by $56 \mathrm{~mm}$, while the SmartRF05EB measures $114 \mathrm{~mm}$ by $128 \mathrm{~mm}$ ). The CC2530 version used in this board is the CC2530F256, with $256 \mathrm{kB}$ of flash memory. The FT232BL chip provides an USB to serial UART interface, allowing the $\mathrm{ZC}$ to send and receive data from the PC. The standard-A USB connector is used for data transfer and to power the ZC. This board also integrates an antenna connector, which is equal to the one used by the CC2530EM, and several other auxiliary components. The total cost of all the components of this board is around 33 euros.

Likewise, for the ZigBee routers (ZR), the TI development boards can be replaced by a much smaller and cheaper CC2530-based module and a power supply. For example, the ANAREN A2530R24CZ1GM module measures $19 \mathrm{~mm}$ by $11 \mathrm{~mm}$ and costs around 19 euros. The prices presented in this paper are retail prices for small quantities. Wholesale prices tend to be lower.

In terms of software, the development platform used for the WSN devices was the Z-Stack version Z-Stack-CC2530-2.5.0, supplied also by Texas Instruments, which supports the profiles ZigBee e ZigBee PRO of the ZigBee 2007 standard. 


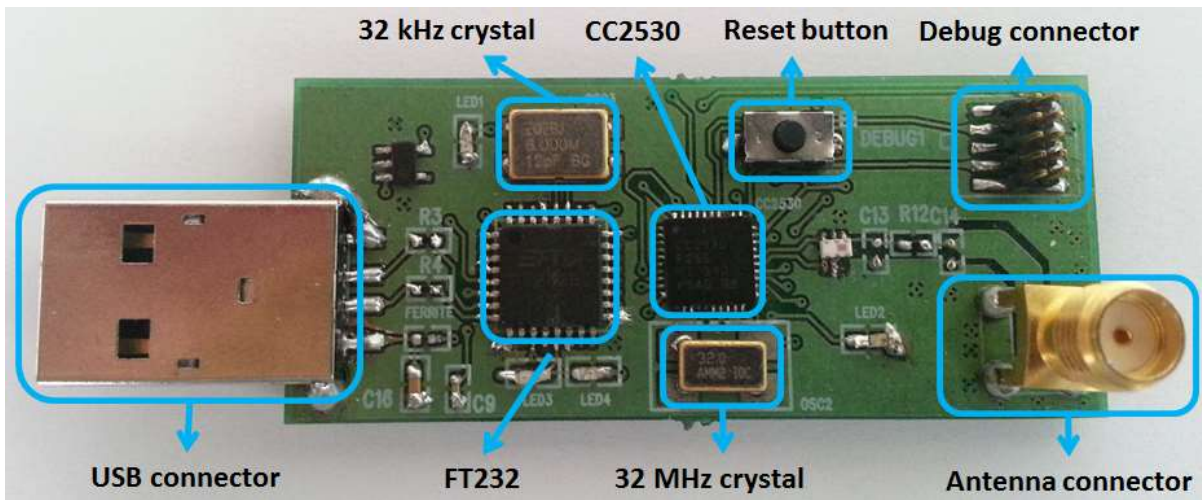

Fig. 3. Developed central station board.

\subsection{Refrigeration equipments}

Regarding the sensors, the analog probe 1 is used to monitor the evaporator temperature and the analog probe 2 monitors the ambient temperature at the refrigeration equipment. When the door is closed, the contact of the door sensor is closed and the digital input P2.1 of the ZR reads a low logic level value, otherwise it reads a high level value.

The actuators of the refrigeration equipment that are controlled by the ZR are the expansion valve and the fan. The output ports of the ZR are not able to provide the voltage or the current required to control these actuators directly (the maximum output current for the P1 port is $20 \mathrm{~mA}$ ). Therefore, the pins P1.0 and P1.1 are connected to relays that are used to control the expansion valve and the fan, respectively.

The refrigeration equipment operates between two predefined temperatures. The stopping temperature is the lower bound temperature desired for the system. When this temperature is reached, the refrigeration must stop. In contrast, the starting temperature is the maximum operating temperature specified for the system. When this temperature is reached, the refrigeration must start. The ZR controls the temperature of the refrigeration equipment through the monitoring of probe 2 , which provides the ambient temperature. When its reading is equal or higher than the starting temperature, the expansion valve is switched on, in order to refrigerate the equipment, until it reaches the stopping temperature.

Once the refrigeration is turned on, the fan is also switched on, except in the following cases: the door sensor is on, which means that the door is open; or the system is defrosting. When the door is opened, both the fan and the expansion valve are switched off. The ZR controls the defrosting process through the monitoring of probe 1, which provides the evaporator temperature.

\subsection{Central station}

The central station is composed by the $\mathrm{ZC}$ and a PC. The PC allows the visualization of the data collected by all the local refrigeration equipments, in real-time, as well as 
the remote control of their operation. The PC receives the readings and sends the commands to the $\mathrm{ZC}$ through a serial port.

The PC application, which provides the graphical user interface (GUI) to the user, was developed using Java and the NetBeans IDE v8.0.2. The data that can be monitored and/or configured on the PC application, for each one of the refrigeration equipments, are the following: equipment number; ambient temperature; evaporator temperature; state of the door sensor; state of the fan; state of the expansion valve; starting temperature; and stopping temperature.

\section{$4 \quad$ Results and Discussion}

This section presents results from experimental field tests performed in an industrial environment, with the purpose of evaluating the performance of the ZigBee network. Each network device was composed by two boards: a SmartRF05EB and a CC2530EM. One ZigBee device was programmed as $\mathrm{ZC}$ and the other devices were programmed as ZRs. These tests were performed in a meat processing factory where there was a constant movement of people and materials.

The placement of the ZC and the ZRs in the factory is shown in Fig. 4. In this configuration, it was noticed that, due to the distance and the propagation conditions, some of the ZRs (1, 2, 4 and 6) were not able to communicate directly with the ZC. Nevertheless, these ZRs were still able to exchange data with the $\mathrm{ZC}$, by taking advantage of the ZigBee multihop capabilities. Therefore, in this deployment scenario, frames from/to ZRs 1 and 2 were routed through ZR 3, while frames from/to ZRs 4 and 6 were relayed by ZR 7 .

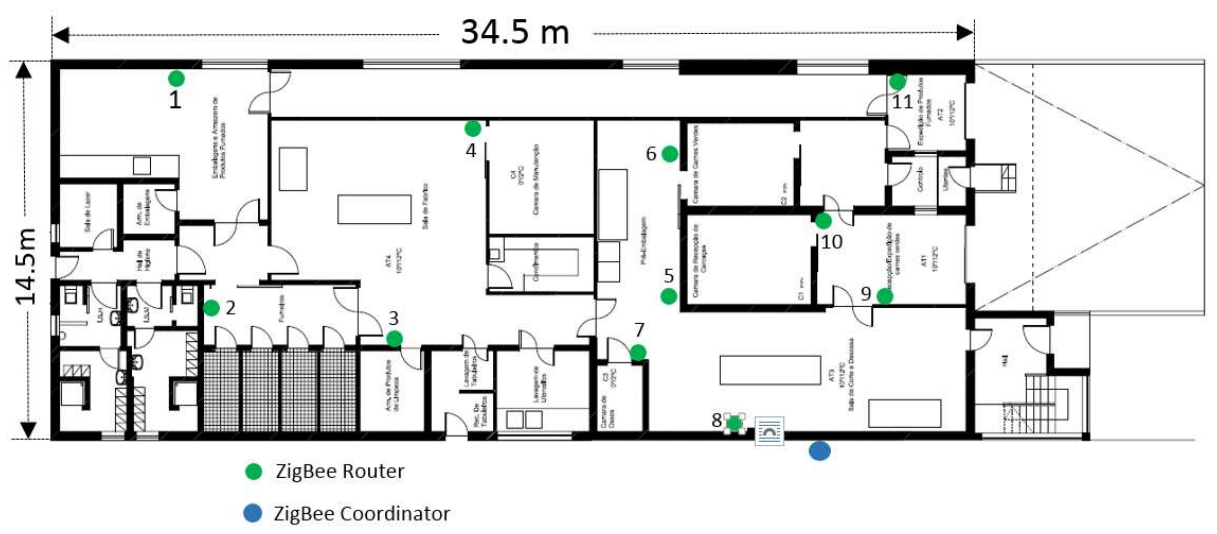

Fig. 4. Distribution of the ZigBee devices in the factory for the tests.

The Wi-Spy 2.4x spectrum analyzer was used to assess the presence of other RF sources in the same spectrum used by the ZigBee devices (the $2.4 \mathrm{GHz}$ frequency band), which may potentially cause interference. This analyzer has shown that the spectrum was being used by two Wi-Fi networks, in channels 4 and 12. The tests presented in 
this paper were performed using two different ZigBee channels, 11 and 26, which are located at the extremities of the available $2.4 \mathrm{GHz}$ band. The carrier frequencies for these channels are $2405 \mathrm{MHz}$ and $2480 \mathrm{MHz}$, respectively. Regarding the co-located Wi-Fi networks, channels 4 and 12 have carrier frequencies of $2427 \mathrm{MHz}$ and $2467 \mathrm{MHz}$, respectively. Taking into account that the bandwidth of Wi-Fi signals is 22 $\mathrm{MHz}$ and that ZigBee signals occupy a bandwidth of $3 \mathrm{MHz}$, it can be concluded that these $\mathrm{Wi}$-Fi and ZigBee signals do not overlap and, consequently, there were no significant interference from Wi-Fi networks during the tests.

In the first test, 1000 data frames with a payload of 90 bytes were sent consecutively with an interval of $200 \mathrm{~ms}$. One goal of this test was to evaluate the worst-case scenario where large packets (close to the maximum length) are transmitted (large packets are more susceptible to channel errors). Fig. 5 shows the packet loss rate (PLR) for each ZR and ZigBee channel in this test. The results for the two channels are very similar. The main differences are related to the location of the ZRs. ZRs 2 and 6, which were more distant from the ZC and had to send data through other ZRs, presented the worse performance, although still acceptable, while other ZRs routers closer to the ZC were able to send all their packets without errors.

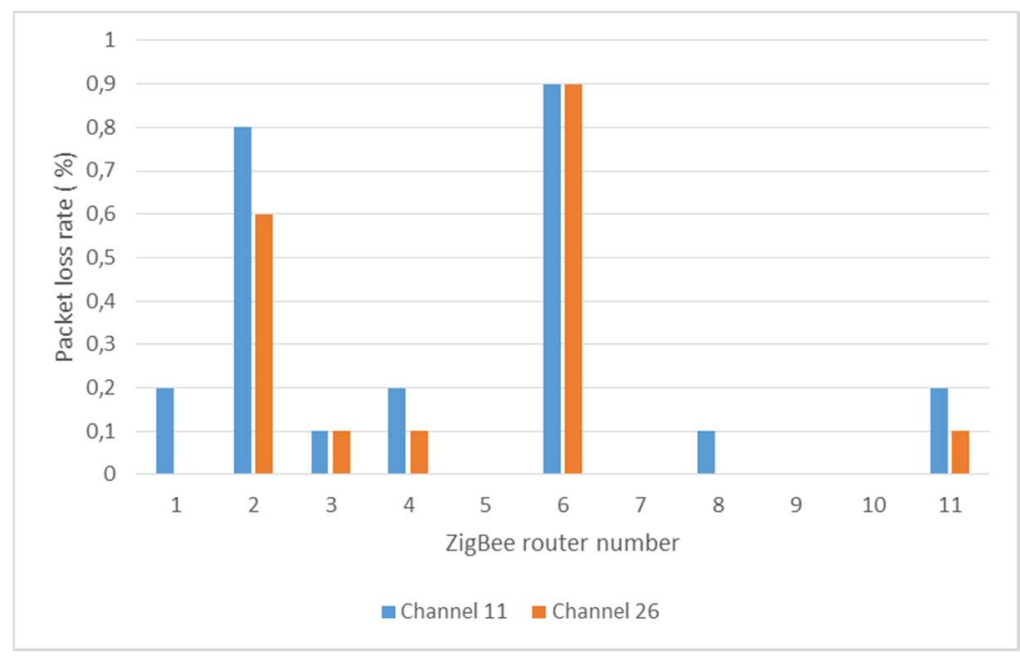

Fig. 5. PLR results with 90-byte payload in different channels.

The second test was performed using the same traffic parameters of the final implementation of the wireless system that is being applied to monitoring and control of refrigeration equipments, which are referred in section 3.1. Therefore, in this test, 1000 data frames with a payload of 20 bytes were sent consecutively with an interval of one second. The test was performed using the channel 26. Fig. 6 presents a comparison between the packet loss rate (PLR) at channel 26, for each ZR, with payload of 90 bytes (first test) and 20 bytes (this second test). From the first to the second test, it can be observed that there is a small decrease in the PLR, on average, which is justified by the smaller payload, which decreases the probability of corruption of the packets. 


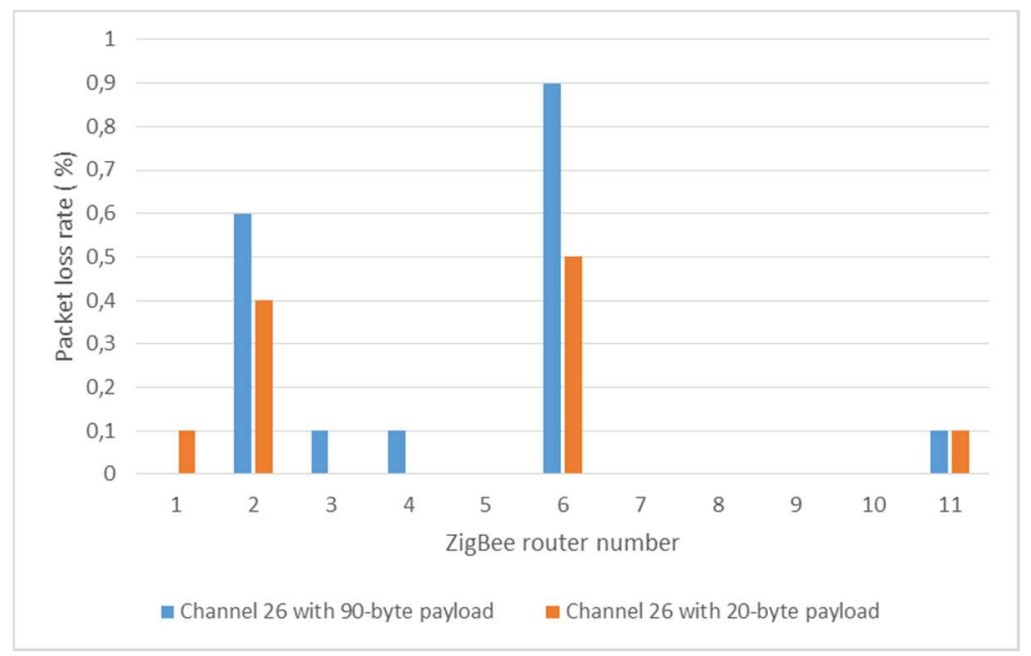

Fig. 6. PLR results with different payload lengths in channel 26.

\section{Conclusions}

This work presents the development of a ZigBee wireless sensor network for monitoring and control of an industrial process, replacing the existing cabled network, based on the LonWorks networking platform. ZigBee routers replaced the local controllers at the refrigeration equipments, while the central management controller was replaced by a ZigBee coordinator and a PC.

The number of industrial/commercial facilities that use refrigeration equipments, namely medium and large commercial areas, has been growing in the last decades. The lifetime of this type of equipments is relatively short, between 10 and 15 years. The consequent required maintenance tasks create an opportunity to replace the legacy wired systems by wireless ones, which presents advantages in terms of deployment and maintenance costs.

Future works may include the provision of more functionalities in the developed system, to allow a more refined control and, consequently, to achieve higher efficiency in the operation of the refrigeration equipments. In this sense, a possible functionality to include is related to the control of the expansion valve, which could have more than two operation levels, being more opened when the temperature of the refrigeration equipment is higher, thus allowing the passing of more refrigeration fluid, or almost closed when the temperature of the refrigeration equipment is near to the stopping temperature. 


\section{Acknowledgements}

This work is supported by FCT (Fundação para a Ciência e Tecnologia) with the reference project UID/EEA/04436/2013, and by FEDER funds through the COMPETE 2020 - Programa Operacional Competitividade e Internacionalização (POCI) with the reference project POCI-01-0145-FEDER-006941.

\section{References}

1. IEEE Std 802.11-2012: Part 11: Wireless LAN Medium Access Control (MAC) and Physical Layer (PHY) Specifications (2012)

2. Kinney, P.: ZigBee Technology: Wireless Control that Simply Works. Communications Design Conference (2003)

3. Borges, L.M., Velez, F.J., Lebres, A.S.: Survey on the Characterization and Classification of Wireless Sensor Network Applications. IEEE Communications Surveys \& Tutorials, 16(4), 1860-1890 (2014)

4. López, H.F., Afonso, J.A., Correia, J.H., Simões, R.: HM4All: A Vital Signs Monitoring System based in Spatially Distributed ZigBee Networks. In: 4th International Conference on Pervasive Computing Technologies for Healthcare (PervasiveHealth 2010), Munich, Germany (2010)

5. Gama, O., Carvalho, P., Afonso, J.A., Mendes, P.M.: Quality of Service in Wireless e-Emergency: Main Issues and a Case-study. In: 3rd Symposium of Ubiquitous Computing and Ambient Intelligence, pp. 95-102 (2009)

6. Zhou, Y. et al. "A design of greenhouse monitoring \& control system based on ZigBee wireless sensor network. In: IEEE International Conference on Wireless Communications, Networking and Mobile Computing (WiCom 2007), Shanghai, China, pp. 2563-2567 (2007)

7. Gungor, V.C., Hancke, G.P.: Industrial Wireless Sensor Networks: Challenges, Design Principles, and Technical Approaches. IEEE Transactions on Industrial Electronics, 56(10) 4258-4265 (2009)

8. Afonso, J.A., Rodrigues, F., Pereira, P., Gonçalves, H., Afonso, J.L.: Wireless Monitoring and Management of Energy Consumption and Power Quality Events. World Congress on Engineering (WCE 2015), London, United Kingdom, pp. 338-343 (2015)

9. Danfoss: Capacity Controller AK-PC 530. Manual (2010)

10. Danfoss: Temperature Sensor type EKS 111. Technical Brochure (2012)

11. Danfoss: Electrically Operated Expansion Valve Types AKV 10. Datasheet (2012)

12. Danfoss: System Manager AK-SM 850. Quick Setup Guide (2013)

13. Echelon Corporation: Introduction to the LonWorks Platform. Version 2.0 (2009)

14. Texas Instruments: CC253x System-on-Chip Solution for 2.4-GHz IEEE 802.15.4 and ZigBee Applications. Datasheet (2010) 